\title{
INVESTIGACIONES
}

\section{Transitando de estudiante a profesor: rearticulaciones en el tercer espacio de una práctica temprana comunitaria}

\author{
The transition from student to teacher: students' negotiations \\ in the third space of an early community practicum
}

\author{
Mirona Moraru ${ }^{a}$ Lucas Ríos Santana ${ }^{b}$ \\ ${ }^{a}$ Universidad Bernardo O’Higgins, Santiago, Chile \\ Correo electrónico: mirona.moraru@ubo.cl \\ ${ }^{\mathrm{b}}$ Universidad Bernardo O`Higgins, Santiago, Chile \\ Correo electrónico: lucas.rios@ubo.cl
}

\begin{abstract}
RESUMEN
El artículo analiza las experiencias de tres estudiantes de tercer año de pedagogía en inglés que asumieron el rol de profesores en el marco de un proyecto que funcionó como una práctica temprana comunitaria; los estudiantes dirigieron talleres de reforzamiento de inglés a un grupo de alumnos de primero a cuarto básico en una comuna con un alto índice de pobreza. Utilizando el concepto de tercer espacio de Homi Bhabha, el artículo explora el impacto de esta práctica en la trayectoria profesional de los estudiantes. Durante la práctica, ellos se encuentran en un tercer espacio, siendo ni estudiantes, ni profesores, sino que algo entre medio. Se argumenta que este espacio hibrido permite una transición parcial de estudiante a profesor, que se manifiesta a través de una serie de negociaciones de tensiones. Por tanto, el artículo analiza las principales rearticulaciones que este proceso genera desde la perspectiva de los estudiantes.
\end{abstract}

Palabras clave: tercer espacio, hibridez, prácticas tempranas comunitarias, transición, educación

\begin{abstract}
The article explores the experiences of three third-year Chilean EFL student teachers who took on the role of teachers in a community engagement project based in Santiago during the first semester of 2017. During this early community practicum, the task of the students was to conduct extracurricular English workshops for a group of pupils coming from disadvantaged backgrounds. Using Homi Bhabha's concept of third space, the article focuses on the impact that this practicum had on the professional trajectory of the students. While on placement, they find themselves in a third space, being neither students, nor teachers, but somewhat in-between. It is argued that this hybrid space entails a partial transition from student to teacher, which manifests itself through a series of tensions that are negotiated during this process. As a result, the article analyses some of their most important beliefs that change as a result of this placement.
\end{abstract}

Key words: third space, hybridity, early community practicum, transition, education 


\section{INTRODUCCIÓN}

El artículo analiza las experiencias de tres estudiantes de tercer año de pedagogía en inglés que asumieron el rol de profesores en el marco de un proyecto de vinculación con el medio de la universidad que imparte la carrera. Se considera que en el contexto de la carrera el proyecto funcionó como un tipo de práctica temprana comunitaria ya que los estudiantes dirigieron talleres de reforzamiento de inglés a un grupo de alumnos de primero a cuarto básico en un colegio ubicado en una comuna de Santiago con un alto índice de pobreza, y por lo tanto, un alto riesgo de vulnerabilidad. Utilizando el concepto de tercer espacio del teórico Homi Bhabha, el artículo explora el impacto de esta práctica en la trayectoria profesional de los estudiantes. Durante esta práctica temprana comunitaria ellos se encuentran en un tercer espacio, siendo ni estudiantes, ni profesores, sino que algo entre medio. Entonces, se argumenta que este contacto con la comunidad educativa representa un espacio hibrido que permite una transición parcial de estudiante a profesor, que se manifiesta a través de una serie de negociaciones de tensiones e disyunciones. Desde esta perspectiva, el presente artículo analiza las experiencias y reflexiones de los estudiantes con un enfoque en las principales rearticulaciones que este proceso genera.

A continuación, el artículo primero da cuenta de la literatura disponible acerca de las prácticas pedagógicas en Chile, con un enfoque en los estudios que exploran las experiencias de los estudiantes. Después, se describe el proyecto, se explica la metodología del estudio y se introduce el marco teórico diseñado por Homi Bhabha, cuya herramienta principal es el concepto de tercer espacio. Luego, se procede al análisis de las experiencias de los estudiantes, con un enfoque en el proceso de transición de estudiante a profesor que el marco de la práctica temprana comunitaria les permite.

\section{PRÁCTICAS PEDAGÓGICAS EN CHILE}

Según Ávalos, las prácticas pedagógicas son entendidas como cualquier actividad en donde el estudiante se encuentre en relación directa con el establecimiento educacional, ya sea en el formato de clases supervisadas o guiadas, o cualquier otro acercamiento (ej. observación, familiarización o intervención) con cualquier centro educativo (Ávalos, 2002: 108). Este autor asegura que el objetivo de dichas prácticas es "permitir la aproximación gradual de los estudiantes al trabajo profesional, y al mismo tiempo facilitarles la construcción e internalización del rol docente" (Ávalos, 2002: 109), por lo que las prácticas pedagógicas permitirían a los estudiantes vincular sus aprendizajes teóricos-prácticos con la realidad expresada en los centros educacionales.

Según un informe del programa de Mejoramiento de la Calidad y la Equidad en la Educación Terciaria (Centro Microdatos, 2017), uno de los desafíos que enfrenta la educación superior en Chile en cuanto a la calidad de la educación va en crear prácticas pedagógicas que sean "más rigurosas y efectivas" (Centro Microdatos, 2017: 7). Por lo tanto, siguiendo a Contreras et al. (2010: 86), sería necesario realizar investigación sobre las prácticas pedagógicas con respecto a qué hacen efectivamente los estudiantes, sus reflexiones sobre lo aprendido, y las condiciones sobre las que se realizan dichas prácticas con el fin de poder repensar el desarrollo de prácticas en las carreras de pedagogía. 
Las investigaciones que podemos encontrar en la literatura sobre prácticas pedagógicas en general en Chile son escasas. Según el estudio realizado por Cisternas (2011), en donde analiza investigaciones en educación en Chile entre los años 1996 y 2007, sólo un $10 \%$ de dichas investigaciones se centran en las prácticas pedagógicas; y la actualidad no dista demasiado de aquel panorama. La mayoría de los estudios referentes a prácticas pedagógicas son sobre las prácticas profesionales o finales ${ }^{1}$, ya sea identificando la eficacia de dichas prácticas (cf. Báez et al., 2015; Cornejo, 2014) o de los profesores supervisores o guías (cf. Cornejo, 2014; Díaz \& Bastías, 2012; Jofré \& Gairín, 2009; Labra, 2011; Latorre, 2009; Montecinos, Barrios \& Tapia, 2011), o exhibiendo las tensiones y desafíos de dichas prácticas finales (cf. Gorichon, Ruffinelli, Pardo \& Cisternas, 2015; Hirmas \& Cortés, 2015; Montecinos \& Walker, 2010). A pesar de esto, según Hirmas \& Cortés (2015), investigaciones posteriores al año 2010 no abarcan únicamente la práctica profesional sino que también las más tempranas en ámbitos como: la relación entre teoría y práctica (cf. Montecinos et al., 2011); las formas de acompañamiento en las prácticas (cf. Solís et al., 2011) o la reflexión y percepción de los estudiantes (cf. Rittershaussen, Contreras, Suzuki, Solís \& Valverde, 2004; Tagle, del Valle, Flores \& Ackley, 2012; Williamson, Abello, Ferreira \& Espinoza, 2015).

Finalmente, aún más escasa es la literatura referente a las percepciones y reflexiones de los estudiantes durante las prácticas pedagógicas. A pesar de esto, ciertos autores, como Bobadilla, Cárdenas, Dobbs y Soto (2009) y Nocetti (2016a) mantienen que es necesario explorar las experiencias de los estudiantes teniendo en cuenta su voz como los actores principales de dichas prácticas. Por lo tanto, hay varios estudios que hacen breve referencia a este aspecto (cf. Bobadilla et al., 2009; Cornejo \& Fuentealba, 2008; Díaz \& Bastías, 2012; Díaz, Sanhueza, Martínez \& Roa, 2010), o exploran más profundamente ciertas reflexiones de los estudiantes en práctica (cf. Barahona, 2014, 2015a, 2015b; Nocetti, 2016a, 2016b; Rittershaussen et al., 2004; Tagle et al., 2012; Williamson et al., 2015).

Uno de los estudios más recientes y relevantes que ha tomado en consideración las reflexiones de los estudiantes de pedagogía es el de Barahona (2014, 2015a), quien investiga si dichas reflexiones van acorde a los objetivos esperados por la universidad y sus docentes. Barahona enfatiza que estas experiencias no sólo sirven para acercarlos a la realidad, sino también hace que "los estudiantes reflexionen y contrasten la realidad de la universidad con la realidad del colegio" (Barahona, 2014: 119). El resultado sería que exista una mayor crítica frente a lo aprendido en la universidad y un mayor conocimiento de cómo se pueden aplicar estos aprendizajes a distintas realidades. No obstante, Barahona (2015a: 79-92) explica que los estudiantes expresan contradicciones entre lo que espera la universidad de ellos y su rendimiento en cuanto a: la aplicación de los conocimientos (los docentes universitarios esperan que los estudiantes apliquen metodologías específicas que no van acorde al contexto); la carga académica (los estudiantes tienen que planificar y desarrollar material para sus prácticas y además rendir en sus tareas en la universidad); y su rol como docentes (la universidad espera que actúen como docentes en la sala de clases de sus prácticas mientras los establecimientos educacionales aún los ven como estudiantes quienes no tienen autonomía completa).

Contreras et al. (2010, pp. 94-97) identifican tres tipos de prácticas dependiendo del momento en el que se realizan dentro del programa de estudios: práctica inicial, intermedia y final. Las prácticas se inician en una instancia de observación y familiarización del contexto; continúan en una instancia intermedia de realización de intervenciones de menor magnitud dentro del aula; y finalizan en una instancia en donde la intervención y la toma de decisiones son mayores. 
En la siguiente sección describimos un proyecto que funcionó como una práctica temprana comunitaria y cuyo impacto en la identidad profesional de los estudiantes es analizado en las próximas secciones en base a las reflexiones de aquellos estudiantes.

\section{DESCRIPCIÓN DEL PROYECTO}

Durante el primer semestre del año 2017, los estudiantes de tercer año de la carrera de Pedagogía en Inglés de una universidad ubicada en Santiago participaron en un proyecto piloto que ofrecía talleres de reforzamiento de inglés. El proyecto se desarrolló en un colegio particular subvencionado de la comuna de Lo Espejo y fue dirigido hacia los alumnos de primero a cuarto año básico.

Es importante mencionar que aunque en el curriculum chileno la enseñanza de inglés es obligatoria empezando desde el quinto año básico (Ministerio de Educación, 2012), el colegio ofrece clases de inglés a todos sus alumnos empezando desde el nivel de pre-kinder. Situado en la segunda comuna con mayor índice de pobreza de Santiago² (Ministerio de Desarrollo Social, 2014), el colegio atiende alumnos en situación de vulnerabilidad socioeconómica; en este contexto, la enseñanza de inglés en edades tempranas está entendida como una herramienta que puede llevar potencialmente a movilidad social y económica. Sin embargo, el colegio recibe alumnos de otros establecimientos quienes no han tenido la oportunidad de aprender inglés. Además, los alumnos descendidos necesitan apoyo adicional para seguir el ritmo requerido. Es hacia este tipo de alumnos que los talleres estuvieron dirigidos, con el fin de nivelar su dominio de inglés y aumentar el nivel de motivación.

El proyecto consistió en varias etapas cuyo objetivo fue apoyar y preparar a los estudiantes para el desarrollo de los talleres de inglés. Primero, se les presentó el proyecto y la metodología de trabajo. Segundo, los estudiantes asistieron a una sesión de inducción por parte de la coordinadora del departamento de inglés del colegio, en donde se familiarizaron con el entorno disciplinar y pedagógico del colegio. Tercero, los estudiantes observaron las clases en dos instancias. Cuarto, el grupo participó en una sesión de reflexión guiada en torno a sus observaciones, en base a la cual hicieron el ejercicio de planificar un simulacro de clase retroalimentada por los coordinadores del proyecto. Finalmente, se desarrollaron los talleres de inglés.

En cuanto a los talleres, es importante mencionar que los alumnos del colegio fueron divididos en dos grupos: primero y segundo año básico (17 alumnos) y tercero y cuarto año básico (19 alumnos). El número total de los estudiantes fue de 22. Dadas ciertas restricciones temporales e impuestas por el colegio, durante los talleres los estudiantes cumplieron distintos roles. Solo tres estudiantes voluntarios cumplieron el rol de profesor en distintos momentos ya que según la coordinadora del departamento de inglés del colegio, el número de profesores tenía que ser limitado, porque los alumnos necesitaban una rutina. El resto de los estudiantes cumplieron el rol de monitores, siguiendo un modelo de rotación voluntaria. Entre las responsabilidades de los monitores fueron el manejo conductual y

Más específico, "el año 2011, se estima que el 21,9\% de la población comunal se encontraba en situación de pobreza lo que corresponde a una tasa mayor a la registrada a nivel regional $(11,5 \%)$ y nacional $(14,4 \%)$ " (Ministerio de Desarrollo Social, 2014: 2). 
apoyo orientado hacia ciertos alumnos con el fin de ayudar al profesor a cumplir sus objetivos. Además, todo el curso fue dividido en grupos encargados de planificar las clases ofrecidas por los profesores y los monitores. Todos los estudiantes, independientemente del rol elegido, tuvieron acceso a los objetivos decididos por la coordinadora del departamento de inglés del colegio.

\subsection{PRÁCTICA TEMPRANA COMUNITARIA}

En el marco de la dicha carrera de pedagogía en inglés se puede considerar que el proyecto funcionó como una práctica temprana comunitaria. Esta perspectiva surge en el marco de tres puntos clave.

Primero, es una práctica temprana desde una perspectiva temporal por su posición en la malla, ya que el primer taller de práctica oficial que ofrece la carrera es en el sexto semestre, mientras que el proyecto se desarrolló un semestre antes. Esto significa que esta fue la primera instancia en que los estudiantes entraron a una sala de clase con un rol distinto al de estudiante.

Segundo, el proyecto también puede ser considerado una práctica temprana desde una perspectiva basada en el conocimiento pedagógico adquirido por los estudiantes antes del proyecto, ya que dichos conocimientos eran básicos. Esto es consecuencia del enfoque de la malla curricular en donde durante los primeros dos años la carrera está enfocada principalmente en la adquisición del inglés; por lo tanto, al comienzo del proyecto, los estudiantes habían tenido solamente dos módulos relacionados a su formación como docentes: Socio-Antropología Educacional y Curriculum $1^{3}$.

Tercero, el proyecto lo consideramos una práctica temprana destacando su distinción con una práctica inicial en donde en su mayoría se realiza un trabajo de observación y reflexión sin participación. Por el contrario, los estudiantes tuvieron la instancia de poder participar activamente dentro del aula, teniendo un rol protagónico. Además, cabe destacar que los estudiantes tenían autonomía completa en los talleres, pero que contaban con apoyo y control de calidad de parte de la universidad y los docentes coordinadores en la etapa de planificación y diseño de clases ${ }^{4}$.

Finalmente, los talleres realizados en el proyecto se enfocaron en proveer herramientas para que distintos estudiantes, en particular los que ingresaban al establecimiento de primero a cuarto básico y los que ya estaban en el colegio, pero que mostraban falencias en la asignatura de inglés, no se vieran desfavorecidos en la adquisición de competencias en el idioma inglés. Por lo tanto, estos talleres que funcionaban a modo de reforzamiento tenían un matiz de ser prácticas comunitarias, especialmente debido a que se dirigían a una comunidad con estudiantes en situación de vulnerabilidad socio-económica.

En resumen, nuestra concepción de práctica temprana comunitaria es entendida así por su temporalidad en la malla curricular, por los conocimientos que han adquirido los estudiantes, por su distinción con una práctica inicial en el rol activo que toman los

Cabe destacar que durante el progreso del proyecto los estudiantes tuvieron los módulos de Psicología Evolutiva, Evaluación Educacional y Curriculum 2.

$4 \quad$ Los estudiantes recibían apoyo de la universidad en la obtención e impresión del material y de los docentes a cargo del proyecto en el diseño y revisión de dicho material teniendo en cuenta que debía cumplir con los objetivos de cada taller y el nivel de los alumnos. 
estudiantes y en su objetivo de proveer apoyo a una comunidad en una situación desfavorable tanto social como económicamente 5 .

En este marco, el objetivo principal del artículo es presentar las experiencias de los estudiantes involucrados en este proyecto y, a través de los patrones encontrados, analizar el potencial impacto de este tipo de prácticas tempranas comunitarias en su formación como profesores de inglés.

\section{METODOLOGÍA}

Dado el enfoque en las experiencias de los estudiantes, la metodología del estudio tiene una orientación cualitativa. Por lo tanto, para poder obtener una perspectiva comprensiva acerca de este proceso recopilamos datos a través de tres tipos de instrumentos: diarios de campo, ensayos reflexivos y entrevistas semi-estructuradas.

Primero, se realizó una observación participante durante todos los talleres impartidos. El propósito fue generar un diario de campo $^{6}$ de los talleres, lo que funcionó como una exploración inicial de esta práctica temprana comunitaria. Segundo, los 22 estudiantes redactaron un ensayo reflexivo acerca de la influencia que tuvo el proyecto en su formación inicial como docentes. Tercero, basado en información recopilada a través de los primeros dos instrumentos, se condujeron entrevistas semi-estructuradas con los participantes clave del proyecto, los tres estudiantes que cumplieron el rol de profesores. Cabe destacar que, dado el enfoque del estudio, el presente artículo está basado principalmente en la información entregada en las entrevistas con los tres estudiantes. El resto de la información fue utilizada como complemento que permitió yuxtaponer y comprobar los temas emergentes, especialmente en las etapas iniciales del análisis.

En la siguiente sección introducimos brevemente el marco teórico cuyos lentes conceptuales nos ayudarán analizar los datos recopilados.

\section{TERCER ESPACIO}

El término de tercer espacio fue propuesto por Homi Bhabha en su búsqueda de una nueva conceptualización para procesos de transformación y cambio cultural, entre otros fenómenos (Rutherford, 1990). Es un concepto que tiene sus raíces en la teoría poscolonial, dado que uno de los enfoques centrales de Bhabha (2002) era analizar los fenómenos sociales y culturales que resultaron de la caída del Imperio Británico en la segunda mitad del siglo XX. Por un lado, estos fenómenos se referían a la composición de la sociedad británica, sus nuevas características, y las identidades emergentes de la (nueva) población inmigrante

\footnotetext{
Nuestra concepción de práctica temprana comunitaria tiene similitudes con el proyecto realizado y evaluado por Williamson et al. (2015) en donde se instaura un proyecto de práctica temprana con servicio voluntario.

6 Según Delamont, la observación participante incluye "una combinación de observación y entrevistas" (Delamont, 2004: 206). Por lo tanto, este método permitió incluir dos perspectivas acerca del mismo proceso. Por un lado, se pudo observar el desarrollo práctico de los talleres, el comportamiento de los alumnos y estudiantes en el aula, los desafíos y las soluciones encontradas. Por otro lado, los estudiantes fueron entrevistados acerca de todos estos aspectos observados para entender sus puntos de vista sobre "lo que están haciendo, pensando y diciendo" (Delamont, 2004: 206).
} 
que empezó a llegar al "país madre" especialmente después del final de la Segunda Guerra Mundial (cf. Bhabha, 2002). Por otro lado, el concepto ha sido utilizado para analizar distintos otros fenómenos relacionados como, por ejemplo, la literatura poscolonial (cf. Bhabha, 2002; Rutherford, 1990). Además, el concepto ha sido extendido por Bhabha mismo para entender la relación entre teoría y práctica (Bhabha, 1988), diferencia cultural (Bhabha, 2002), o cambios políticos (Bhabha, 1988), entre otros.

Entonces, el concepto de tercer espacio surge principalmente de la necesidad de entender tanto procesos de transformación cultural (por ejemplo, el caso de las comunidades inmigrantes), como la relación entre entidades usualmente conceptualizadas como polarmente opuestos (por ejemplo, la relación entre teoría y práctica). Con la noción de tercer espacio, Bhabha trata de eludir la "política de polaridad" (Bhabha, 2002: 59) y el "binarismo cultural" (Rutherford, 1990: 211), sugiriendo la posibilidad de una articulación entre las dos entidades en cuestión.

Además de esta articulación, él afirma que, en realidad, las dos entidades constituyentes de cualquier binarismo propuesto (ya sea dos culturas distintas, dos idiomas, o dos posiciones de poder) nunca representan posiciones fijas (Rutherford, 1990: 210-211). Más bien, estas dos posiciones o formas culturales pueden ser identificadas como tal, pero hay que recordar que se encuentran en un "continuo proceso de hibridez" (Rutherford, 1990: 211) que "rechaza el esencialismo de un original previamente establecido, o de una cultura originaria" (Rutherford, 1990: 211).

Tomando en consideración la hibridez previa de cada una de las dos culturas (u objetos, o posiciones), el encuentro entre estas dos manifestaciones genera un tercer espacio (Bhabha, 1988, 2002; Rutherford, 1990). Entones, el tercer espacio, o la hibridez, se refiere al desarrollo de posiciones, formas culturales, identidades, $u$ otras entidades que nacen como una novedosa articulación entre los dos supuestos binarios en cuestión. En las palabras de Bhabha,

la hibridez [o el tercer espacio] soporta las huellas de esos sentimientos y prácticas que la informan, igual a una traducción ... la hibridez pone junto las huellas de ciertos otros significados y discursos. No les da la autoridad de ser previos en el sentido de ser originales: son previos solamente en el sentido de ser anteriores. El proceso de hibridez cultural genera algo diferente, algo nuevo e irreconocible, un área nueva de negociación de significado y representación (Rutherford, 1990: 211).

Por lo tanto, el tercer espacio se refiere esencialmente a algo nuevo, tangible o intangible. Siguiendo a Moje et al. (2004), el tercer espacio es la posibilidad de desafiar y remodelar los dos supuestos binarios hacia nuevas alternativas, prácticas, discursos, o formas de conocimiento. Se trata de una transformación entendida por Bhabha (2002: 39-60, 273; Rutherford, 1990: 211) como una forma de traducción y de negociación de tensiones y disyunciones que hace posible la generación de nuevas posiciones, que no son "ni Uno, ni Otro sino algo más, inter-medio" (Bhabha, 2002: 264).

Puesto que la propuesta de Bhabha abarca un tema tan amplio como el de transformación, el concepto de tercer espacio ha sido extendido y utilizado en una variedad de disciplinas. Dado el enfoque de nuestro artículo, en lo siguiente introducimos brevemente los estudios más importantes del ámbito educativo que se proponen entender las prácticas pedagógicas utilizando el tercer espacio como lente conceptual. 


\subsection{TERCER ESPACIO, EDUCACIÓN Y PRÁCTICAS PEDAGÓGICAS}

En el ámbito educativo, algunos de los estudios más significativos que emplean la noción de tercer espacio analizan prácticas de alfabetización ${ }^{7}$ (ver, por ejemplo, Gutiérrez, 2008; Gutiérrez, Baquedano-López \& Tejeda, 1999; Moje et al., 2004), la negociación de conocimiento entre la casa y la escuela (Moje et al., 2004), o entre la casa y la universidad (Greca, 2016).

En el ámbito específico de formación inicial docente, el concepto de tercer espacio ha sido utilizado para explorar el tema de las prácticas pedagógicas desde la perspectiva de dos problemáticas estrechamente relacionadas: la relación entre escuela y universidad y la relación entre teoría y práctica (ver, por ejemplo, Cuenca, Schmeichel, Butler, Dinkelman \& Nichols, 2011; Elsden-Clifton \& Jordan, 2015; Forgasz, Heck, Williams, Ambrosetti \& Willis 2018; Grenfell, 1998; Jordan \& Elsden-Clifton, 2014; Martin, Snow \& Torres, 2011; McDonough, 2014; Trent, Gao \& Gu 2013; Zeichner, 2010). Este enfoque ha surgido debido a una crítica extensa hacia la desarticulación entre la formación teórica universitaria y la realidad práctica escolar. Como resultado, la literatura suele ocupar el concepto de tercer espacio para explorar distintas estrategias de articulación entre los dos ámbitos. Una de las estrategias más recurrentes es la misma creación de un tercer espacio en la forma de una colaboración innovadora entre la universidad y los centros de práctica con el fin de que se equilibren tanto las relaciones de poder entre los docentes universitarios y los profesores del centro de práctica, como el tipo de conocimiento adquirido por los estudiantes (ver Cuenca et al., 2011; Grudnoff, Haigh \& Mackisack, 2017; Martin et al., 2011; Zeichner 2010).

Mientras la perspectiva de la creación de un tercer espacio como una colaboración innovadora es atractiva, conceptualmente este artículo se aleja de ella hasta un cierto punto. Primero, en el presente estudio entendemos cualquier relación entre dos entidades como un tercer espacio, sin la necesidad de que sea una colaboración especial o innovadora; entonces, entendemos cualquier tipo de práctica pedagógica como un tercer espacio. Segundo, y estrechamente relacionado con el primer punto, justamente porque los terceros espacios "ya existen en todas partes como un elemento de la condición humana" (Bruna, 2009: 222), estamos de acuerdo con las dudas expresadas por Bruna (2009) con respeto al enfoque en la "creación" de un tercer espacio en lugar de un enfoque en el análisis que puede potenciar este concepto. En este sentido, preferimos restringir el uso de la noción de tercer espacio a su potencial de lente conceptual que nos permite "visibilizar las conexiones y los movimientos" entre las posiciones inhabitadas por los estudiantes en práctica (Jordan \& Elsden-Clifton, 2014: 223).

Al mismo tiempo, mientras la mayoría de los estudios están enfocados en el binario universidad - escuela, el objetivo de este estudio es explorar la transición parcial de estudiante a profesores a través de una práctica temprana comunitaria. De manera similar a Gannon (2010) y Sinner (2010), nos concentramos en un punto potencialmente clave de la formación identitaria de los futuros profesores que se encuentran en un tercer espacio, es decir, en una posición intermedia, hibrida, entre ser estudiante y ser profesor. Cabe destacar que utilizamos el tercer espacio como un concepto que, como Jordan \& ElsdenClifton (2014) comentan, ofrece "un marco para reconocer las tensiones y los dilemas de

Usualmente estas prácticas son relacionadas al empoderamiento de los estudiantes marginalizados. 
los estudiantes negociando terrenos desconocidos" (Jordan \& Elsden-Clifton, 2014: 223). Entendiendo esta práctica temprana comunitaria como el comienzo de un "rito de paso profesional" (Gannon, 2010: 21), el articulo explora la visión de los mismos estudiantes acerca de esta transición, con un enfoque en la negociación de sistemas de valor divergentes, la reconfiguración identitaria y la reinterpretación de lo que es ser profesor.

\section{ANÁLISIS: ENTRE SER ESTUDIANTE Y PROFESOR}

La presente sección propone el análisis de las entrevistas con los tres estudiantes que asumieron el rol de profesores durante la práctica temprana comunitaria. Además, el análisis está complementado por información recopilada a través de las otras fuentes de información mencionados en Sección 4. El análisis propuesto está dividido en dos partes principales. Primero, introducimos las bases que permiten el desarrollo de un espacio hibrido, entre ser estudiante y profesor. Segundo, ya que "el proceso de hibridación es acompañado de crítica, ruptura y desafíos hacia las ortodoxias establecidas" (Grenfell, 1998: 20), nos enfocamos en la negociación de transiciones, rearticulaciones y (auto-) traducciones experimentadas por los estudiantes durante este proceso.

\subsection{LAS BASES DE LAS REARTICULACIONES: EL CAMBIO DE ROL}

Siguiendo las experiencias de los estudiantes, se puede argumentar que las bases para las rearticulaciones experimentadas por los estudiantes tienen un denominador común: el cambio de su rol de estudiantes. Este cambio toma lugar progresivamente; en una primera instancia los estudiantes observan las clases, mientras que en una segunda instancia asumen el rol de profesor en los talleres. En las siguientes sub-secciones detallamos las consecuencias de estos cambios de rol en la construcción de un tercer espacio.

\subsubsection{Estudiantes-observadores}

El proceso de observación es sumamente importante para los estudiantes ya que esta es una primera instancia que permite un cambio de perspectiva acerca de su propia identidad:

sólo el hecho de estar en una sala ya a uno le cambia la idea de lo que está estudiando (Isabel)

aunque uno salió no hace mucho del colegio y sabe cómo funciona, estar dentro de la clase con otro rol es diferente, entonces uno como que se va formando una [idea distinta sobre esta] realidad (Andrea).

Como observadores, los estudiantes entran en un espacio donde todas las relaciones de poder con las que ya están acostumbrados y cuales definen su identidad sufren modificaciones. Por lo tanto, ellos no son ni estudiantes, ni profesores; más bien, se encuentran en una posición intermedia desde donde pueden reflexionar acerca de los procesos observados, relacionándolos tanto con su propia experiencia como exalumnos, como con sus expectativas como futuros profesores. Desde esta posición, Isabel se 
concentra no necesariamente en la organización de los contenidos de la clase, sino en el manejo de clase de la profesora y en la relación que construye con sus alumnos:

más que ir a observar cómo se hacía una clase ... yo quería prestar atención en cómo ella manejaba los tiempos, cómo manejaba los estudiantes y cómo se desenvolvían los estudiantes con respecto a lo que ella enseñaba ... me llamó la atención su forma de manejar 40 niños de 5 años (Isabel).

Similarmente, el proceso de observación le permite a Andrea pensar en la multitud de niveles que un profesor necesita manejar simultáneamente:

me cuestioné al principio si era lo que yo realmente quería porque me pareció muy complicado ... el ejercicio que yo hice cuando fui a observar es verme adelante, yo traté de pensar, si yo estoy haciendo esta clase ¿me funcionaría? ... dije no, esto es demasiado complicado para mí, así lo vi (Andrea).

Enfrentando la realidad del aula desde una posición que no es ni de alumna, ni de profesora, Andrea se imagina a sí misma como profesora. Dándose cuenta de la complejidad de la tarea, ella también empieza a reflexionar acerca de algunas de sus ideas más enraizadas, e incluso cuestiona la misma decisión de ser profesora.

Mientras este primer contacto con el aula como observadores representa una base incipiente para algunas reflexiones e inquietudes, la próxima sub-sección sostiene que el reconocimiento y autorreconocimiento que reciben los estudiantes en el rol de profesor en los talleres es el elemento más importante que permite la construcción de un tercer espacio, es decir, una posición intermedia entre ser estudiante y profesor. A su vez, esto genera una serie de transiciones e rearticulaciones fundamentales de los entrevistados.

\subsubsection{Estudiantes-profesores: reconocimiento y autorreconocimiento}

Para Isabel, Andrea y Diego, asumir el rol de profesor representa un cambio de posición fundamental; dictar los talleres de inglés no involucra solamente enseñar, sino también un reconocimiento simbólico de su posición como profesores en los ojos del alumnado, del establecimiento, y de su propia perspectiva.

Es interesante notar que el proceso de reconocimiento de nuestros estudiantes como profesores por parte de los alumnos no fue siempre directo, sino que pasó por una serie de negociaciones:

al principio [los niños] no nos tomaban en cuenta ... un día la profesora [del curso] tuvo que conversar con ellos y decirles que nosotros somos profesores y que nosotros les hacemos las clases y ahí de a poquito empezaron a poner más atención pero al principio nos costaba bastante (Andrea).

Dado que inicialmente los alumnos no reconocían a Andrea en su rol de profesora, negándose a obedecerla, la profesora del curso intervino como agente de negociación entre el grupo y la nueva profesora, otorgándole poder. Al mismo tiempo, Andrea empezó a negociar su reconocimiento por parte de los alumnos tratando no de cambiar el contexto, 
sino tratando de ella misma adaptarse a la realidad del aula, así como vemos en la Sección 6.2.1.

Por otro lado, la experiencia de Isabel demuestra la alternativa donde la nueva profesora está reconocida sin necesidad del mismo nivel de negociación:

el hecho de ser profesora a una le da cierta autoridad porque al final los niños a uno lo ven como el profe ... no dimensionan la diferencia entre ser estudiante y profesor de inglés, si uno es el profe, es el profe y se acabó, entonces el hecho de ser profe a uno le da esa autoridad que uno usa a su favor para hacer callar, para poner reglas, y para enseñar (Isabel).

Se puede argumentar que este tipo de reconocimiento funciona como un puente en la posición que ocupan los estudiantes tanto en frente de sus alumnos, como en relación a su trayectoria profesional. Esto es así por el poder que ellos adquieren en frente de los alumnos.

Aparte de los alumnos, los estudiantes también fueron reconocidos como profesores por parte del establecimiento:

en el colegio nos hacen sentir parte de la comunidad educativa ... allá en el colegio nos tratan como profe, allá en el colegio somos profe ... a uno no lo miran como estudiante en práctica, a uno lo miran como profe (Andrea).

nosotros éramos como un profesor más ahí, todos nos saludaban como si fuéramos parte del colegio ... las autoridades del colegio nos dieron absoluta libertad ... nosotros ahí hacíamos y deshacíamos o sea éramos los profesores (Isabel).

Es importante subrayar que la institución hace que los estudiantes se sientan parte de la comunidad educativa en una posición de relativo poder, es decir, como pares de los otros profesores. Esto se lleva a cabo a través de dos estrategias: por un lado, el tratamiento que los estudiantes reciben de parte del establecimiento fuera del aula es el que reciben los mismos profesores, y por otro lado, adentro de las clases, los estudiantes tienen un nivel muy elevado de autonomía, similar al de los profesores del colegio. En consecuencia, los estudiantes no son vistos como alumnos o, incluso, como estudiantes en práctica, donde el establecimiento es responsable por su desempeño. Por el contrario, las relaciones de poder se balancean gracias a que los talleres sirven como apoyo para el colegio y la comunidad. Esto es notable ya que hace que los estudiantes se empoderen de su nuevo rol y lleguen a un cierto nivel de autorreconocimiento como profesores:

lo que más me gustó [fue] tener la oportunidad de sentirse profe ... [el proyecto nos da la] libertad de poder creernos los profes, de ser profes, de yo ir y yo hago mi clase con mis estudiantes (Andrea).

Comoyahemosmencionado, consideramosqueelreconocimientoy autorreconocimiento como profesores es el elemento clave que genera un tercer espacio en la trayectoria de los futuros profesores, permitiéndoles pasar por una serie de rearticulaciones fundamentales para su identidad profesional. 


\subsection{REARTICULACIONES HACIA UNA TRANSICIÓN DE ESTUDIANTE A PROFESOR}

Estar en frente de sus alumnos, negociando y recibiendo el reconocimiento y autorreconocimiento como profesores significa para Isabel, Andrea y Diego encontrarse en un tercer espacio que les permite rearticular una serie de ortodoxias establecidas y que da pie a su transición de estudiante a profesor. En concreto, nos enfocamos en tres rearticulaciones clave que los estudiantes enfatizan en las entrevistas: el rol del profesor, el ambiente de aprendizaje, y su rol de estudiante en la universidad durante y después del proyecto.

\subsubsection{El rol de profesor}

El contacto con la realidad del colegio y con los alumnos en el nuevo rol de profesor involucra la re-construcción de lo que significa la labor docente desde la perspectiva de nuestros estudiantes:

me [di] cuenta del papel que tiene el profe en la sala, que no es solamente una persona que transmite información, sino que va más allá de eso (Isabel).

[aprendí que] ser profe no es estudiar y saberse los contenidos, ser profe es un montón de cosas ... es pararse adelante a hacer una clase, preocuparte de que tus alumnos aprendan, considerar a los apoderados (Andrea).

aprendí que si la familia no está involucrada, que si un niño no posee los recursos no delimita que ese niño desarrolle sus capacidades al máximo, siento que está en el docente explotar y reconocer fortalezas y motivar al estudiante a superarse a sí mismo ... entonces siento que realmente aprendí a motivar eso en mi estudiante (Diego).

A pesar de su larga trayectoria como alumnos y estudiantes interactuando con profesores diariamente, es principalmente en el encuentro con la comunidad educativa desde esta nueva posición que los entrevistados empiezan a reflexionar seriamente acerca del rol multidimensional del profesor. En particular, ellos enfatizan la importancia del conocimiento pedagógico por encima del conocimiento disciplinar. Además, se dan cuenta del rol complementario que tienen los profesores, entregando valores, motivando, y apoyando al alumno emocionalmente. En el caso de alumnos en riesgo de vulnerabilidad, esto se convierte en una responsabilidad mayor, donde el profesor y el establecimiento representan la única fuente de apoyo académico y, potencialmente, el principal referente valórico. Los entrevistados también destacan el rol del profesor como lazo entre la escuela y la familia y la necesidad de poseer las herramientas necesarias para negociar esta relación.

\subsubsection{Manejo de aula y metodología}

Otro aspecto que los tres entrevistados enfatizaron fue la problemática del manejo del aula y la metodología que utilizaban en los talleres. Para empezar, los tres señalan sus bajas expectativas tanto de los niños, como de sí mismos antes de empezar los talleres:

pensé que iban a ser niños conflictivos ... que íbamos a tener que partir por tratar de poner disciplina... también yo pensé que no iba a poder sobrellevar la situación en 
caso de que se escapara de las manos ... la primera vez que yo fui iba súper nerviosa, yo no sabía cómo hacer clases, nunca había estado en frente de niños así en una sala real, entonces mis expectativas respecto a mí eran también bajas porque yo decía, no sé metodología, sé lo básico, ¿cómo lo voy a hacer? (Isabel).

al principio me dio un poco de miedo ... yo siempre tuve miedo de cómo manejar el orden de la sala ... fue un desafío que no sabíamos cómo hacer [la clase] porque estábamos todos conscientes de que el nivel es distinto, ellos se aburren más fácil entonces hay que mantenerlos ocupados y eso nos costaba a nosotros ¿cómo lo hago? (Andrea).

Desde su rol de estudiantes, los entrevistados tenían bajas expectativas acerca de su capacidad de estructurar de manera efectiva el ambiente de aprendizaje, especialmente en términos del manejo disciplinario de aula. Esto puede ser consecuencia de que no hayan tenido ramos relacionados a este aspecto como mencionamos anteriormente. Sin embargo, una vez en el rol de profesor, ellos reflexionan acerca de la relación entre el manejo efectivo del aula y la metodología empleada ya que ven una conexión entre el comportamiento de sus alumnos y las didácticas que utilizan.

Las expectativas de los estudiantes se cumplieron parcialmente puesto que el manejo de clase representó uno de los desafíos más significantes que ellos recalcan de los primeros talleres:

[el mayor desafío] fue mantener el manejo de la clase [especialmente en] mi primera sesión de profesor (Diego).

lo que sí me costó al principio fue manejar el class management, el orden, de que pongan atención, de que estén tranquilos (Andrea).

Sin embargo, dada la duración del proyecto y la cierta libertad de tomar decisiones, los entrevistados tuvieron la oportunidad de encontrar formas de negociar su rol de profesor, experimentar distintas estrategias para obtener un ambiente de aprendizaje efectivo, y así lograr superar sus bajas expectativas:

fueron las 3 primeras semanas que funcionó así desordenado y cuando me di cuenta en qué estaba fallando quizás yo, porque también habían problemas de que yo no sabía cómo manejar a los niños, una vez que descubrí el problema y le encontré la solución, ahí ellos también respondieron de la manera esperada (Andrea).

Esto representa un proceso de descubrimiento y autodescubrimiento, dado que empiezan a reflexionar acerca de sus debilidades y potenciales soluciones.

Por un lado, los entrevistados quiebran una ortodoxia establecida acerca de la dinámica del aula donde el profesor sólo entrega información y debe ser estricto para mantener el orden de la sala:

al principio [era estricta] y después empecé a darme cuenta que eso no funcionaba y empecé a probar que pasaba si a una niña le dejaba que repartiera las guías, la niña se motivaba ... uno va entiendo que ellos quieren ser partícipes de una forma u otra de la sala de clases entonces si uno los deja con ciertos límites ellos van a participar y van a responder (Isabel). 
a ellos les gusta que ... no solo esté yo explicando sino que les gusta interactuar (Andrea).

lo que aprendí a ver [es] la proactividad del estudiante ... aprendí a interactuar con los estudiantes ... aprendí que el alumno mismo tiene que ser el que maneja su educación entonces yo voy a ser un guía más que un sabelotodo en el aula (Diego).

Desde su nueva posición, los entrevistados se dan cuenta de que la dinámica profesoralumno no es necesariamente vertical y, una vez rearticulada está relación, empiezan a experimentar distintas otras modalidades de relacionarse con los alumnos. Además, ellos también descubren que no es sólo el profesor el que construye significado en el aula, sino que el proceso de enseñanza-aprendizaje es más bien una negociación y una co-construcción entre el profesor y los alumnos.

En la misma línea, los estudiantes rearticulan la relación entre el conocimiento que tienen que entregar y las formas de entregarlo:

como futura profesora [entendí] lo importante [que es] la forma de enseñar más allá de lo que enseñamos, cómo lo enseñamos, porque hay varias formas o sea, yo puedo enseñar el verbo to be de una forma súper aburrida pero si yo llevo eso a algo que a ellos les gusta probablemente voy a captar el 100\% de su atención ... estuve pensando siempre qué puedo hacer ahora para que les guste, qué video les puedo mostrar para que se entretengan, pero a la vez aprendan (Isabel).

Desde su posición de profesora, Isabel reflexiona en torno a la variedad de metodologías que se pueden utilizar y cómo las distintas formas de enseñar influyen la construcción del ambiente de aprendizaje. Además, yendo aún más allá, ella también demuestra preocupación por lograr un proceso de enseñanza-aprendizaje significativo, que tenga consecuencias fuera del taller que imparte.

\subsubsection{De vuelta a la universidad}

Es importante subrayar que haber asumido el rol de profesor en los talleres de inglés también genera una serie de rearticulaciones con respecto al rol de estudiante al cual nuestros entrevistados regresan durante y después del proyecto.

Primero, ellos manifiestan haber transitado hacia un enfoque más reflexivo acerca del conocimiento entregado en la universidad:

uno se vuelve más reflexivo porque si me enseñan, no sé, el verbo to be, ya, me lo tengo que aprender porque yo sé que algún día los niños me van a preguntar por qué hay que usar el verbo to be y voy a tener que tener una respuesta, en eso me enfoco un poco más, en dar respuestas, por ejemplo, no sé, tía por qué hay que ponerle "s" al verbo en tercera persona ... entonces como ahí me estoy como perfeccionando un poco más ... [estoy tratando de] ser un poco más reflexiva en términos del por qué usar tal cosa en vez de más que usarlo (Isabel).

Haber tenido la oportunidad de entender la realidad del aula desde el rol de profesor, la relación con el proceso formativo de los estudiantes sufre modificaciones, ya que los 
entrevistados no regresan al rol más tradicional de estudiante, donde reciben e internalizan información. Por el contrario, ellos están más conscientes de su rol como futuros profesores, donde no tienen que meramente repetir, sino que aplicar el conocimiento adquirido durante su proceso formativo. Entonces, para ellos aprender adquiere otro significado, donde el conocimiento teórico tiene una relevancia en el aula, en la práctica. Además, conociendo parcialmente la realidad del aula y algunos de los retos del ser profesor, ellos están ahora más preparados para construir junto con sus propios docentes este proceso formativo. Es decir, están en una posición que les permite negociar su formación a través de un nivel más elevado de participación activa, reflexiva, crítica y más informada en base a la realidad educativa actual.

Segundo, y estrechamente relacionado con el primer punto, los estudiantes también adquieren una perspectiva más crítica acerca del programa de pedagogía en inglés que están estudiando, especialmente acerca de la relación entre teoría y práctica:

yo considero que la malla de aquí en términos de dominio del inglés está súper bien pero falta como el tema de class management y ese tipo de cosas, yo creo que [esa] es un área un poco más débil y después del proyecto uno descubre de que falta (Andrea). una sugerencia a la malla es el tema de la práctica un poco antes ... porque siento que tener práctica en un colegio recién en cuarto año, o sea tres años después de haber visto inglés 123456 hasta el 7, mucho inglés, poca metodología, cero práctica (Isabel). yo no poseo todo el arsenal disponible para llevar a cabo una buena clase entonces siento que en ese sentido está como mal planteada la malla ... siento que el tema del dominio del inglés aunque sí es relevante creo que es más vital las capacidades como docente ... porque uno puede saber todas las cosas del inglés pero si no sabe cómo enseñar no va a ser profesor (Diego).

Los estudiantes reflexionan acerca de las herramientas con las cuales la universidad los ha equipado hasta el punto del proyecto. Desde la perspectiva del cambio de rol que experimentaron, ellos comentan críticamente el enfoque en el conocimiento disciplinar de la malla curricular que guía su proceso formativo en los primeros dos años. Cabe recordar que esta fue una práctica temprana comunitaria que no forma parte oficialmente del curriculum y no está alineada al resto del programa, por lo cual los estudiantes percibieron un nivel elevado de disyunción entre su formación y la responsabilidad de asumir el rol de profesor en un aula real. Sin embargo, ya que la carrera asumió este riesgo, lo más significativo es que se produjo una rearticulación de parte de los estudiantes en cuanto a la relación teoría-práctica, lo cual genera cambios importantes en la forma de asumir el rol de estudiante como futuro profesor, como hemos visto en el punto anterior. Además, esta rearticulación demuestra un nivel alto de compromiso crítico con el programa, lo cual lleva hacia una co-construcción del proceso formativo potencialmente más efectiva.

\section{CONCLUSIÓN}

Hemos visto que el cambio de rol de estudiante a profesor durante los talleres de inglés ha sido clave para una reconfiguración radical de los entrevistados en torno a aspectos que influyen en la formación de su identidad profesional. El siguiente testimonio sirve para entender las dimensiones de esta reconfiguración: 
antes del proyecto yo me veía como estudiante de pedagogía, yo estaba aquí aprendiendo inglés, aprendiendo cómo ser profe y listo yo decía, yo salgo y me dedico a ser profe pero con el proyecto lo veo mucho más cercano, ya no veo el ser profe así como lejos, ahora es como que estoy siendo profe (Andrea).

En otras palabras, esta instancia de práctica temprana comunitaria sirvió como un tercer espacio donde los entrevistados pudieron comenzar su transición de estudiante a profesor a través de una serie de rearticulaciones y rupturas de las ortodoxias establecidas en su rol de estudiantes. Como hemos visto, en el caso de nuestros sujetos, las ortodoxias establecidas rearticuladas se refieren principalmente a las expectativas acerca de sí mismos en el rol de profesor, al rol de profesor, a la manera de establecer un ambiente de aprendizaje efectivo, a su rol de estudiante de pedagogía y el programa que cursan. El resultado de ubicarse en este espacio hibrido que les permitió la negociación de tensiones y disyunciones es la posibilidad de involucrarse en un proceso de reconstrucción identitaria radical. A su vez, esta experiencia los lleva un poco más cerca del ser profesor en el continuum estudianteprofesor, o tal como dice Andrea, les permite "estar siendo" profesores.

Para concluir, conviene subrayar que tanto la experiencia de la práctica temprana comunitaria, como los aspectos explorados en el presente artículo representan sólo una parte de la transición de estudiante a profesor. En consecuencia, es importante seguir explorando este proceso de transición con un enfoque en las experiencias de los estudiantes. El significado de este tipo de investigaciones es que nos permiten entender tanto las negociaciones de los estudiantes con los centros de práctica, como su co-construcción de su proceso de formación profesional en la universidad. Esto representa un aspecto clave a la hora de negociar cambios en las mallas curriculares de las carreras de pedagogía con el fin de mejorar las relaciones entre teoría y práctica, y universidad y centros de práctica.

\section{REFERENCIAS BIBLIOGRAFICAS}

Ávalos, B. (2002). Profesores para Chile, Historia de un proyecto. Santiago de Chile: Ministerio de Educación.

Báez, M., Casas, M., del Valle, R., Fonseca, G., Jara, E., Lagos, J., ...Viveros, R. (2015). La formación práctica en educación. Tres miradas a los sistemas de práctica en la formación inicial docente. En Centro Interuniversitario de Desarrollo, CINDA (Eds.), La formación práctica en la universidad y su impacto en el perfil de egreso (pp. 37-102). Santiago de Chile: CINDA.

Barahona, M. (2014). Pre-service teachers' beliefs in the activity of learning to teach English in the Chilean context. Kul'turno-istoricheskaya psikhologiya - Cultural-Historical Psychology, 10(2), 116-122.

Barahona, M. (2015a). Contradictions in the activity of learning to teach English in Chile. In B. Selau $\&$ R. Fonseca de Castro (Eds.), Cultural Historical Approach: Educational Research in different contexts (pp. 73-99). Porto Alegre: EDIPUCRS.

Barahona, M. (2015b). English language teacher education in Chile: A cultural historical activity theory perspective. Abingdon: Routledge.

Bhabha, H. K. (2002). El lugar de la cultura. Buenos Aires: Manantial. . (1988). The commitment to theory. New Formations, 5, 5-23.

Bruna, K. (2009). Jesús and María in the jungle: an essay on possibility and constraint in the thirdshift third space. Cultural Studies of Science Education, 4, 221-237. doi: 10.1007/s11422-0089159-0 
Bobadilla, M., Cárdenas, A., Dobbs, E. \& Soto, A. M. (2009). "Los rodeos de la práctica". Representaciones sobre el saber docente en el discurso de estudiantes de pedagogía. Estudios Pedagógicos, 35(1), 239-252.

Centro Microdatos. (2017). Proyecto: "Percepción de los empleadores en cuanto a la calidad y pertinencia de los graduados/titulados de las instituciones de educación superior que cuentan con el apoyo del MECESUP 3". Informe Final. Facultad de Economía y Negocios Universidad de Chile. Recuperado el 18 de junio de 2018 desde http://www.mecesup.cl/usuarios/MECESUP/ File/2017/publicaciones/nacionales/EstudioEmpleadoresBIRFCMD.pdf

Cisternas, T. (2011). La investigación sobre formación docente en Chile. Territorios explorados e inexplorados. Calidad en la Educación, 35, 131-164.

Contreras, I., Rittershaussen, S., Montecinos, C., Solís, M. C., Núñez, C. \& Walker, H. (2010). La escuela como espacio para aprender a enseñar: Visiones desde los programas de formación de profesores de educación media. Estudios Pedagógicos, 36(1), 85-105.

Cornejo, J. (2014). Prácticas profesionales durante la formación inicial docente: Análisis y optimización de sus aportes a los que aprenden y a los que enseñan a aprender "a enseñar". Estudios Pedagógicos, 40(1), 239-256.

Cornejo, J. \& Fuentealba, R. (Eds.). (2008). Prácticas reflexivas para la formación profesional docente: ¿Qué las hace eficaces?. Santiago de Chile: Ediciones UCSH.

Cuenca, A., Schmeichel, M., Butler, B. M., Dinkelman, T. \& Nichols Jr., J. R. (2011). Creating a "third space" in student teaching: Implications for the university supervisor's status as outsider. Teaching and Teacher Education, 27, 1068-1077. doi: 10.1016/j.tate.2011.05.003

Delamont, S. (2004). Ethnography and participant observation. In C. Seale, G. Gobo, J. Gubrium \& D. Silverman (Eds.), Qualitative Research Practice (pp. 205-2017). London: SAGE Publications.

Díaz, C. \& Bastías, C. (2012). Una aproximación a los patrones de comunicación entre el profesor mentor y el profesor-estudiante en el contexto de la práctica pedagógica. Educación XX1, 15(1), 241-263.

Díaz, C., Sanhueza, M. G., Martínez, P. \& Roa, I. (2010). Las concepciones pedagógicas de estudiantes en práctica profesional respecto de su rol en la enseñanza y aprendizaje del inglés en el nivel secundario de enseñanza. CONSENSUS, 15(1), 9-23.

Elsden-Clifton, J. \& Jordan, K. (2015). What's up DOCC? Creating third space courses in teacher education. Artículo presentado en la conferencia the Annual Conference of the Australian Teacher Education Association (ATEA), Darwin, Australia. Recuperado de https://atea.edu.au/ wp-content/uploads/2016/03/atea-2015-elsden-clifton-jordan.pdf

Forgasz, R., Heck, D., Williams, J., Ambrosetti, A. \& Willis, LD. (2018) Theorising the third space of professional experience partnerships. In J. Kriewaldt, A. Ambrosetti, D. Rorrison \& R. Capeness (Eds.), Educating Future Teachers: Innovative Perspectives in Professional Experience (pp. 3347). Singapore: Springer Singapore.

Gannon, S. (2010). Service learning as a third space in pre-service teacher education. Issues in Educational Research, 20(1), 21-28.

Gorichon, S., Ruffinelli, A., Pardo, A. \& Cisternas, T. (2015). Relaciones entre formación inicial e iniciación profesional de los docentes. Principios y desafíos para la formación práctica. Cuaderno de Educación, 66, 1-20.

Greca, M. (2016). Supporting pre-service elementary teachers in their understanding of inquiry teaching through the construction of a third discursive space. International Journal of Science Education, 38(5), 791-813. doi: 10.1080/09500693.2016.1165892

Grenfell, M. (1998). Border-Crossing: Cultural hybridity and the rural and small schools practicum. Artículo presentado en la conferencia the Annual Conference of the Australian Association for Research in Education (AARE). Adelaide, Australia. Recuperado de https://files.eric.ed.gov/ fulltext/ED446881.pdf

Grudnoff, L., Haigh, M. \& Mackisack, V. (2017). Re-envisaging and reinvigorating school-university 
Estudios Pedagógicos XLIV, $\mathrm{N}^{\circ}$ 3: 317-335, 2018

TRANSITANDO DE ESTUDIANTE A PROFESOR: REARTICULACIONES EN EL TERCER ESPACIO DE UNA PRÁCTICA TEMPRANA COMUNITARIA

practicum partnerships. Asia-Pacific Journal of Teacher Education, 45(2), 180-193. doi: 10.1080/1359866X.2016.1201043

Gutiérrez, K. (2008). Developing sociocritical literacy in the third space. Reading Research Quarterly, 43(2), 148-164. doi: 10.1598/RRQ.43.2.3

Gutiérrez, K., Baquedano-López, P. \& Tejeda, C. (1999). Rethinking diversity: Hybridity and hybrid language practices in the third space. Mind, Culture, and Activity, 6(4), 286-303. doi: 10.1080/10749039909524733

Hirmas, C. \& Cortés, I. (2015). Estado del arte. Investigación sobre formación práctica en Chile: tensiones y desafíos. Santiago de Chile: Organización de Estados Iberoamericanos para la Educación, la Ciencia y la Cultura - OEI.

Jofré, G. \& Gairín, J. (2009). Formación inicial de los docentes de enseñanza media. Una mirada desde las universidades «pedagógicas» chilenas. EDUCAR, 44, 31-46.

Jordan, K. \& Elsden-Clifton, J. (2014). Through the lens of third space theory: Possibilities for research methodologies in educational technologies. In S. Zvacek, M. T. Restivo, J. Uhomoibhi $\&$ M. Helfert (Eds.), Proceedings of the $6^{\text {th }}$ International Conference on Computer Supported Education (CSEDU-2014) Volume 1 (pp. 220-224). doi: 10.5220/0004792402200224

Labra, P. (2011). Construcción de conocimiento profesional docente: el caso de la formación en la práctica. (Tesis inédita de doctorado). Universidad Academia de Humanismo Cristiano, Santiago de Chile. Recuperado el 18 de junio de 2018 desde http://bibliotecadigital.academia.cl/ bitstream/handle/123456789/679/Tesis\%20completa.pdf?sequence=1\&isAllowed=y

Latorre, M. (2009). Prácticas pedagógicas en la encrucijada: Argumentos, lógicas y razones de los actores educativos. Revista Pensamiento Educativo, 44-45, 185-210.

Martin, S., Snow, J. \& Torrez, C. (2011). Navigating the terrain of third space: Tensions with/in relationships in school-university partnerships. Journal of Teacher Education, 62(3), 299-311. doi: $10.1177 / 0022487110396096$

McDonough, S. (2014). Rewriting the script of mentoring pre-service teachers in third space: Exploring tensions of loyalty, obligation and advocacy. Studying Teacher Education, 10(3), 210221. doi: 10.1080/17425964.2014.949658

Ministerio de Desarrollo Social. (2014). Reporte comunal: Lo Espejo, Región Metropolitana. Observatorio Social.

Ministerio de Educación. (2012). Decreto 439. Recuperado el 18 de junio de 2018 desde http://bcn. $\mathrm{cl} / 1 \mathrm{uxth}$

Moje, E., Ciechanowski, K., Kramer, K., Ellis, L., Carrillo, R. \& Collazo, T. (2004). Working toward third space in content area literacy: An examination of everyday funds of knowledge and discourse. Reading Research Quarterly, 38(1), 38-70. doi: 10.1598/RRQ.39.1.4

Montecinos, C., Barrios, C. \& Tapia, M. F. (2011). Relación entre estilos de supervisión durante la práctica profesional y las creencias de autoeficacia de los estudiantes de pedagogía en educación básica. Revista Perspectiva Educacional, 50(2), 96-122.

Montecinos, C. \& Walker, H. (2010). La colaboración entre los centros de práctica y las carreras de pedagogía. Docencia, 42, 64-73.

Montecinos, C., Walker, H., Rittershaussen, S., Nuñez, C., Contreras, I. \& Solís, M. C. (2011). Defining content for field-based coursework: Contrasting the perspectives of secondary preservice teachers and their teacher preparation curricula. Teaching and Teacher Education, 27, 278-288. doi: 10.1016/j.tate.2010.09.001

Nocetti, A. (2016a). Experiencia de reflexión en estudiantes de pedagogía en educación media en biología y ciencias naturales en las asignaturas de práctica pedagógica y profesional en una universidad de la región del Bío Bío, Chile. (Tesis inédita de doctorado). Universitat de Barcelona, Barcelona. Recuperado el 18 de junio de 2018 desde http://diposit.ub.edu/dspace/ bitstream/2445/102037/1/ANdlB_TESIS.pdf

Nocetti, A. (2016b). Facilitadores de reflexión docente durante la práctica profesional en escuelas 
vulnerables de Concepción, Chile. EDUCADI, 1(1), 41-54.

Rittershaussen, S., Contreras, I., Suzuki, E., Solís, M. C. \& Valverde, P. (2004). Prácticas iniciales en la formación de educadores. Pensamiento Educativo, 35, 192-215.

Rutheford, J. (1990). The third space. Interview with Homi Bhabha. In J. Rutheford (Ed.) Identity: Community, Culture, Difference (pp. 207-221). London: Lawrence and Wishart.

Sinner, A. (2010). Negotiating spaces: The in-betweeness of becoming a teacher. Asia-Pacific Journal of Teacher Education, 38(1), 23-37. doi: 10.1080/13598660903474171

Solís, M. C., Núñez, C., Contreras, I., Rittershaussen, S., Montecinos, C. \& Walker, H. (2011). Condiciones de la formación práctica de los futuros profesores. Estudios Pedagógicos, 37(1), 127-147.

Tagle, T., del Valle, R., Flores, L. \& Ackley, B. (2012). Las creencias de autoeficacia percibida de estudiantes de pregrado de pedagogía en inglés. Revista Iberoamericana de Educación, 58(4), $1-12$.

Trent, J., Gao, X. \& Gu, M. (2014). Language teacher education in a multilingual context: Experiences from Hong Kong. The Netherlands: Springer Netherlands.

Williamson, G., Abello, E., Ferreira, S. \& Espinoza, F. (2015). Formación inicial docente: Prácticas tempranas con servicio voluntario. Revista Electrónica Diálogos Educativos, 29(15), 90-105.

Zeichner, K. (2010). Rethinking the connections between campus courses and field experiences in college-and-university-based teacher education. Journal of Teacher Education, 61(1-2), 89-99. doi: 10.1177/0022487109347671 
